Lengua y Sociedad, revista de lingüística teórica y aplicada

Vol. 17, n. ${ }^{\circ}$ 2, Lima, julio-diciembre de 2018, pp. 139-161 https://doi.org/10.15381/lengsoc.v17i2.22356

\title{
De la causalidad a la súplica: nuevas funciones del marcador pues
}

\section{From causation to appeal: new functions of the marker pues}

\author{
Verónica Jenny Lazo García \\ Departamento Académico de Lingüística \\ CILA-FLCH-UNMSM \\ veronica.lazo@unmsm.edu.pe
}

\begin{abstract}
Resumen
Este artículo describe las funciones del marcador pues en el español hablado en Lima. Se considera la relación entre el contexto lingüístico con el tono y la duración. Se asume que las funciones discursivas del mencionado marcador se organizan en un continuum semántico de continuidad-causalidad-argumentación-contacto-intersubjetividad, lo cual posibilita su empleo en diferentes discursos (narraciones, entrevistas o diálogos). Finalmente, se muestra cómo la posición final predomina en el registro espontáneo (géneros narrativo y conversacional); la posición inicial, en el registro semiespontáneo (con valores expresivos de súplica, sarcasmo, regaño y desgano); y la posición intermedia, en el registro semiformal (género entrevista).
\end{abstract}

Palabras clave: Marcador discusivo pues; registro lingüístico; género discursivo; función pragmática; parámetros acústicos.

\begin{abstract}
The functions of the discursive marker pues in Spanish spoken in Lima is described in this article. The relationship between the linguistic context with pitch and duration is considered. It is assumed that the discursive functions of the mentioned marker are organized in a semantic continuum of continuity-causality-argumentation-contact-intersubjectivity, which makes it possible to use them in different discourses (narrations, interviews or dialogues). Finally, it is shown how the final position predominates in the spontaneous register (narrative and conversational genres); the initial position, in the semi-spontaneous register (with expressive values of supplication, sarcasm, scolding and reluctance); and the intermediate position, in the semi-formal register (gender interview).
\end{abstract}

Key words: Discussion marker pues, linguistic registration, discursive genre. pragmatic function, acoustic parameters. 


\section{Introducción}

Se han documentado diversos usos de pues, tanto en la modalidad escrita (conector causal y consecutivo -sobre todo formal-) como en la oral (comentador, metadiscursivo). En las interacciones de los limeños ${ }^{1}$, se aprecia nuevas construcciones donde aparece esta partícula, las cuales cumplen diversas funciones pragmáticas. Además, aunque la naturaleza de pues puede resultar ambigua o imprecisa para los investigadores (Villa Frey, 2017), los enunciados donde aparece resultan fácilmente interpretables para los hablantes, quienes lo emplean en ciertas construcciones más o menos fijas dentro de posiciones específicas en el discurso, con formas fonológicas propias y con específicas funciones pragmáticas.

La presente investigación busca describir cómo se organizan las funciones pragmáticas de la partícula pues en los discursos espontáneos del español de Lima.

\section{Metodología}

La muestra está constituida por 3 mujeres y 3 varones, quienes fueron grabados, con consentimiento, en una entrevista (2016). Además, se ha considerado dos conversaciones espontáneas dentro de la casa de la que realiza la presente investigación, una realizada el 6 de julio de 2014 y otra de 22 de abril de 2016, (con un total de 5 minutos con 81 segundos). Por último, se elicitó 30 diálogos semiespontáneos juveniles (2018).

\section{Antecedentes. Las funciones de pues}

Delimitar las funciones de las palabras no es sencillo, y mucho menos de partículas como pues. En un primer momento Bello (1887) propone el sentido básico de [causalidad] para pues. Alarcos (1994), por su parte, señala que la [continuidad] es el sentido primario de pues, por lo que postula que puede ser elidido en oraciones yuxtapuestas ilativas. Sin embargo, desde un enfoque pragmático pues no es un simple expletivo, debido a que aporta más que una información ilativa, es decir, puede alcanzar nuevos sentidos para los hablantes según el contexto vía subjetivización. A continuación, enlistamos las funciones de pues propuestas por las 
De la causalidad a la súplica: nuevas funciones del marcador pues

investigaciones de Martín Zorraquino y Portolés (2001), Dorta y Domínguez (2001), Uribe Mallarino (2005), Grajales (2011), Zavala (2006) y Villa Frey (2017).

\subsection{Conjunción causal / conector semántico 0 argumentativo}

Alarcos (1994), Martín Zorraquino y Portolés (1999, 2001) sostienen que la partícula precede a la idea que es causa de la anterior. Grajales (2011) señala que esta función causal se encuentra principalmente en la escritura y no tanto en el lenguaje oral, ya que requiere más planificación. Según Alarcos, la primera oración termina en cadencia e impone una pausa antes de pues. Por ejemplo:

(1) Yo me metí corriendo en la cama para hacerme el dormido, pues me daba miedo preguntarle nada. (Alarcos, 1994, pág. 368)

(2) E: ¿Y usted qué piensa de eso? //

I: (...) y que se lleve esa buena imagen que uno ve de ella / y que la repartan por toda Colombia / por todo el mundo / pues es bueno que / que vean con buenos ojos a la ciudad de uno // (Grajales, 2011)

\subsection{Conjunción / conector consecutivo / conector semántico 0 argumentativo}

Según Bello (1887) y Alarcos (1994), esta conjunción aparece en construcciones ilativas. El sentido suele ser ilativo, consecutivo, continuativo proviene de los contenidos sucesivos de cada oración. Martín Zorraquino y Portolés $(1999,2001)$ afirman que este conector es tónico, evita la posición final del miembro en el que se incluye va delimitado por la entonación y es seguido de pausa. Grajales (2011) señala que al igual que la conjunción causal, el conector consecutivo pues se encuentra principalmente en la escritura y no tanto en el lenguaje oral.

(3) ¿Tantas razones no os convencen? Apelemos pues a los hechos. (Bello, 1887, pág. 359).

(4) ¿Ven ustedes este santurrón? Pues hasta vende hostias [...] y cera. Ingresó, pues, hace siete años en el seminario. (Alarcos, 1994: pág. 323) 
En el registro espontáneo, Dorta y Domínguez (2001) señalan que la función conectora aporta no solo organización sino argumentación, porque expresa consecuencia y valida un comentario anterior. Guevara (2015) sostiene que este pues se ubica siempre en posición central y le da continuidad a la enunciación del locutor. Señala, además, que se puede permutar por entonces, $y$ muestra instrucciones al destinatario para que pueda elaborar sus conclusiones. Según Villa Frey (2017), esta función está estrechamente vinculada a uno de los valores esenciales de pues, específicamente, al valor inferencial o conclusivo.

(5) H1: ¿Has ido alguna vez a revisar un examen? [Risas]

H2: Aún no he hecho ninguno. Bueno, en sociología sí que hice, pero como [risas] no estudiaba pues tampoco hacía falta revisar. (Dorta y Domínguez, 2001)

\subsection{Función narrativa}

Villa Frey (2017) considera que pues se usa con función narrativa cuando el hablante cuenta una historia, anécdota o experiencia personal y este marcador le permite hilar la historia que está narrando, haciendo evidente la conexión entre los enunciados de su discurso. El siguiente ejemplo presenta un caso semejante:

(6) [Ale cuenta sobre un servicio de entrega a domicilio] 1- Ale: ya bueno te sigo contando,

2- Ale: entonces esté:: el chico

3- Ale: entonces subimos pues, el chico lo subió todo

4- Ale y como que de ahí vimos que tenía un paquetazo

5- Ale: entonces yo le dije a mi mamá

6- Ale: oye, no deberías comprar los paquetes grandes, o sea

En el extracto anterior Ale utiliza pues a final de la unidad de habla y este elemento permite vincular los enunciados de las líneas 1 a 3 de la narración. Los enunciados de las líneas 2 y 3 están entrecortados, el uso de pues genera la conexión requerida para continuar con la anécdota que se narra. La autora menciona un patrón recurrente con respecto al uso narrativo en el que el hablante utiliza la frase ya pues, que involucra además las variantes y ya pues, así como entonces, ya pues (o ya pues, entonces). 
De la causalidad a la súplica: nuevas funciones del marcador pues

( 7 )

1- Ale: yo iba a llevar un cuestionario, pero dije por si acaso

2- Ale: y justo había llevado el de ( )

3- Jan: siempre hazlo, sí

4- Ale: sí, sí, ahora siempre lo voy a hacer, y ya pues

5- Ale: y que bueno que me vine con eso ( ) en la mano

6- Ale: y me cayó reco::ntra bien y (.) como que llegamos

7- Ale: y la pobre (.) o sea ni siquiera fue a- al baño na:da

8- Jan: nada?

9- Ale: o sea como que de frente se sentó conmigo =

10- Ale: [y mi mamá se sentó en el sofá

11- Jan: [así?]

12- Ale: y ya pues de ahí le hice Dos cuestionarios larga::zos

13- Ale: y encima de los más grandes, me entiendes?

14- Jan: ya, claro (Co_20160617_MyH)

En (7) Ale utiliza dos veces la frase y ya pues con función narrativa; en ambos momentos (líneas 4 y 12) el marcador sirve para continuar con la anécdota que está narrando e introduce información nueva que conecta con el discurso anterior.

\subsection{Función inferencial}

Según Villa Frey (2017), la proposición que contiene pue se deduce de lo que Corina ha contado previamente, es decir, el contenido se infiere del discurso antecedente.

(8) [Corina habla sobre dos de sus nietos menores]

1- Corina: parecen mellizo los dos

2- Corina: pero se trompean DU:ro, puÑEte se meten =

3- Corina: y la otra que es Más gorda (.) PA le mete así:: (.)

4- Corina: el otro día viene mi nieto con un martillo =

5- Corina: casi le reviente su carita a la:

6- Rosa: o:::h

7- Corina: y él tenía el martillo y decía =

8- Corina: No, ese es mío, no, ese es mío

9- Corina: $y$ - e::- mi nieta JAla el martillo =

10- Corina: y con el martillo PA le dio en la carita al bebe = 


\section{Verónica Jenny Lazo García}

11- Corina: casi la revienta su carita (.) Asu se privó =

12- Corina: y la otra estaba asustada pue (.)

(9)

1- Gabi: entonces mi mamá quería que

2- Gabi: yo la Única hija mujer

3- Gabi: se llame Betsabé como su tíza favorita

4- Gabi: la cosa es que mi papá (.) se va a inscribirme =

5- Gabi: en el (.) en la municipalidad pues cuando yo nazco

\subsection{Marcador comentador / función organizadora}

En primer lugar, Según Portolés, Martín Zorraquino y Uribe, se trata del uso más frecuente en el discurso oral. Presenta su miembro como un comentario nuevo con respecto del discurso que lo precede.

(10)

- ¿Usted conocía a Soleiro?

- ¿Un cojo que iba a Mondoñedo al mercado de Quendas?

_ ¡El mismo! Pues se le apareció a la mujer en figura de cuervo.

$(11)$

-Bueno, pues ahora viene... mm, la parte más descriptiva.

Descríbeme tu lugar favorito, tu habitación, un sitio que te guste...

(Dorta \& Domínguez , 2001, pág. 45)

Para Dorta y Domínguez (2001), en esta función pues le permite al hablante organizar su discurso; por ello recibe el nombre de organizadora. Esta función se asemeja a la función de ganar tiempo para hablar o retrasar la comunicación, propuesta por Uribe (2005) y la función metadiscursiva de Grajales (2011).

Como otros marcadores, crea una función interpersonal entre hablante y oyente, implicando su interacción y aportando cohesión y coherencia a la conversación. Entre sus funciones se encuentran tomar el turno de palabra (Uribe, 2005); señalar un refuerzo argumentativo, es decir, puede añadir una respuesta que confirma lo que se está informando (Portolés y Martín Zorraquino, Uribe, 2005): 
De la causalidad a la súplica: nuevas funciones del marcador pues

(12)

- ¿A qué se dedica?

-Pues él es maestro y escribe algunos libros y da pláticas, conferencias y mi abuela murió hace un par de años.

(13)

— ¿Y después de tantos años todavía te gusta la gramática?

-Pues sí.

Puede añadir una respuesta que da una información no preferida por el interlocutor o poner de relieve el contraste de un enunciado con lo dicho anteriormente:

(14)

A: Juan es una magnífica persona. (Intervención iniciativa)

B: $\uparrow$ Pues a mí no me lo parece. (Intervención reactiva no preferida > marcador contraargumentativo)

\subsection{Marcador aclarador / función reformuladora}

Función confirmativa

Zavala (2006) propone el pues evidencial confirmativo, ya que cuando se produce una pregunta sobre una afirmación y esta pregunta exige la confirmación o la aclaración de lo que se ha dicho anteriormente, la segunda respuesta se enuncia con pues. Villa Frey (2017) también describe esta función en el castellano limeño, con cuatro patrones: sí pues, ya pues, claro pues y así pues. Según Dorta y Domínguez (2001), Zavala y Villa Frey (2017) el marcador ejerce la función de aclaración:

(15)

1- Virginia: Y tus padres, tus abuelos, ¿también vivían en la comunidad?

2- Pedro: Sí

3- Virginia: ¿En esa misma?

4- Pedro: Sí, en esa misma pe (confirmación)

(16) [Manuel cuenta que un amigo que trabaja en una empresa proveedora de tecnología le ha vendido una memoria externa a precio de remate] 1- Manuel: lo reportó como averiado y ya::: me lo dio 
2- Pablo: a::su::

3- Manuel: y ya::: lo puse en mi disco pe (.)

4- Manuel: es que como él ve la parte de garantía

5- Manuel: todo eso (.) ya::

6- Pablo: o sea, lo dio de baja y ya

7- Manuel: lo dio de baja pue (.) ajá

4- Manuel: es que como él ve la parte de garantía

5- Manuel: todo eso (.) ya::

6- Pablo: o sea, lo dio de baja y ya

7- Manuel: lo dio de baja pue (.) ajá

Según Grajales, pues ejerce funciones de ejemplificación, corrección, paráfrasis o recapitulación.

Paráfrasis

(17)

E: ¿Qué es lo que más te gusta de la casa?

I: Mi cuarto / mi cuarto me gusta mucho / porque está en la parte de atrás / entonces de pronto se siente menos ruido que / que en los otros cuartos / y / porque / mi casa es muy iluminada / pues le entra mucha luz / entonces no se necesita casi prender / focos / y mi cuarto me gusta mucho por eso...

Ejemplificación del elemento discursivo anterior

(18)

E: ¿Cómo cree que puede ser su vida si se casa?

I: Si tenemos hijos / que no sea más de uno / o dos máximo / y / que no se me limite / el tiempo para / hacer lo que me gusta / pues / para trabajar / o para salir / o para / no sé / para hacer lo que me gusta / así me imagino yo casada...

\subsection{Función de control de contacto}

La función de control de contacto, según Grajales (2011), es de tipo expresivo-apelativa o fática y su cometido es regular la interacción entre los participantes de la conversación a través de la verificación del contacto con el interlocutor y de 
llamadas de atención que mantienen, renuevan o comprueban la relación con los oyentes (mire, fíjese, hombre). En segmentos narrativos, acompaña un imperativo. En las narraciones en las que aparece esta función de pues, los enunciados son emitidos por un personaje que tiene una superioridad de poder, o una relación cercana con el interlocutor, que le otorga el contexto; se utiliza el marcador discursivo pues para intensificar el imperativo.

(19)

E: ¿y qué piensa del servicio que presta?

// I: súper bueno / porque imagínate pues esa gente en un bus cuánto no se demoran para bajar al centro / en cambio allí bajan / se ahorran los pasajes y todo /

(20)

E: ¿Alguna vez le han atracado / o conoce la historia de alguien que le hayan robado? //

I: una vez casi sí / que / que andaba yo con mi novio / y estábamos pues / con / con el vino / y que tales / y estaba ya tardecito en el centro / y dos de esos / dos malandros pues / hablaban ahí como / como murmurando / que hágale pues / que hágale pues / entonces claro / yo le metí la mano a mi novio / y salimos fue corriendo mejor / pero no nos pasó nada //

Esta función guarda relación con el pues exhortativo (Villa Frey, 2017). Según esta autora, el hablante utiliza la función exhortativa para formular órdenes, pedidos y sugerencias, así como para expresar ruegos o súplicas, que exhortan al oyente a realizar alguna acción específica. Esta función ocurre únicamente en la serie de conversaciones coloquiales.

(21)

1- Manuel: oe, no tienes otra silla para mí?

2- Pablo: te traigo?

3- Manuel: traite una pe

4- Pablo: y ahora?

5- Manuel: traite pe

6- Pablo: normal?

7- Manuel: sí, tráetela normal 
8- (Pablo se va a traer una silla)

Los ejemplos de esta función exhortativa evidencian la similitud existente con el uso de pues a final del enunciado en el español peninsular del s. XVI y XVII. Considérese los siguientes fragmentos:

(22)

1- Elisa: ¡Leonor, Leonor! Quítame este manto luego y escóndele.

2- Elisa: ¡Acaba, pues!

(1632-1634; Tirso de Molina, Los balcones de Madrid; CORDE, cit. en Olbertz 2013: 22)

(23)

1- Acario: Llama ya en essa puerta.

2- Gargullo: ¿Que llame? Espera, pues.

(1545; Lope de Rueda, Comedia llamada Medora; CORDE, cit. en Olbertz 2013: 21)

La estructura con el pues pospuesto comenzó a caer en desuso a partir del s. XVIII en España. El hallazgo de que pues se usaba a final del enunciado en los s. XVI y XVII es relevante para nuestro estudio, ya que indicaría que esta estructura fue difundida por los españoles quienes mantenían con la población indígena un tipo de contacto caracterizado por las órdenes y mandatos breves (Olbertz 2013). Especialmente en zonas rurales, el español andino -reconocido como una variedad arcaizante que tiende al empleo de estructuras sintácticas del español clásico (De Granda 2001)- habría mantenido el uso de construcciones castizas que con el tiempo dejaron de usarse en el español peninsular.

\subsection{Función enfática}

Según Guevara (2015), el marcador fuerza al argumento. No admite ser conmutado. Se encuentra siempre en posición final, la cual permite una conclusión definitiva y enfática con respecto al primer argumento presentado.

(24) [Manuel y Pablo trabajando con la laptop] 1- Manuel: pantalli::ta: cuál pantallita, oe? (risas)

2- Pablo: el ce erre: 
De la causalidad a la súplica: nuevas funciones del marcador pues

3- Manuel: eso se llama wiN

4- Manuel: la tecla Windows wiN

5- Pablo: pero la figura pue::s:

6- Manuel: ya:: ya: ya: pantallita (Co_20160902_HyH)

\section{Aproximación de las funciones de pues}

A partir de los datos presentados, proponemos una aproximación de las funciones discursivas de pues, las cuales se asocian por semejanza de familia con las funciones prototípicas de continuidad, causalidad, argumentación y contacto fijadas por frecuencia y se expresan mediante parámetros acústicos y estructuras discursivas.

Tabla 1. Aproximación de las funciones de pues

\begin{tabular}{l|l|l|l}
\hline \multicolumn{1}{c|}{ Nivel } & Función discursiva & \multicolumn{1}{|c|}{ Código } & \multicolumn{1}{c}{ Posición } \\
\hline \multirow{2}{*}{ Oracional } & Causal y & A & Entre cláusulas \\
& consecutiva & B & Antecede al enunciado conclusivo \\
\multirow{2}{*}{ Relato } & Continuativa e & C & o parte importante del relato \\
\hline \multirow{3}{*}{ Conversación } & inferencial & D & Al Inicio de la intervención \\
\cline { 2 - 4 } & Comentador & E & \\
& Aclarativo, & F & Al final de la intervención. \\
& de contacto & G & \\
\hline & enfático & H & \\
\hline
\end{tabular}

En distintos registros del habla espontánea y en distintos grupos sociales, pues alcanza diversas formas y funciones: puede finalizar respuestas afirmativas con cordialidad o desgano; puede finalizar información dubitativa, afirmativa, corroborativa, aclarativa, obvia o sarcástica; puede presentar una idea concluyente o de resumen en una narración; puede expresar una consecuencia lógica $y$, por último, puede iniciar y terminar enunciados exhortativos. En suma, los sentidos de pues, se agrupan en el continuum [continuidad-causalidad-argumentación-contacto-intersubjetividad]. 


\subsection{Uso de pues en conversación espontánea}

En (25) se aprecia una conversación, en la que el interlocutor C emplea el «pe» aclarativo, con una información obvia. Luego el interlocutor R emplea la construcción «claro pe» con un valor enfático.

$(25)$

$1 \mathrm{R} 1$ een ocho mes a él le han lavao la cabeza

$1 \mathrm{R} 1$ o compare que es, cojudo asegúrate de una vez, ya que $\$$

${ }_{1} \mathrm{C} 1$

$\S$ A él ah,

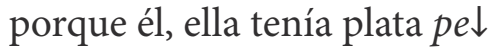

2R2 [iClaro!]

$\mathrm{CH}_{1}[\mathrm{mmm}]$

${ }_{1 \mathrm{C}} 1$ un grupo/ musical/ en apogeo

$\mathrm{R}_{3}$ [shhh]

${ }_{1 \mathrm{C} 1}$ Por eso se ha casado él

${ }_{3} \mathrm{R}_{4}$ ¡Claro pe $\downarrow$ y al toque la ha llenado otra vez!

${ }_{2} \mathrm{C}_{2} ¡ \mathrm{Ah}$ !

En las figuras 1 y 2 se muestra sombreado el contorno tonal descendente de las construcciones que contienen pues con un valor aclarativo y enfático en conversaciones espontáneas. Además, la posición de la partícula es final.

Figura 1. Contorno tonal de la frase Ella tenía plata pe

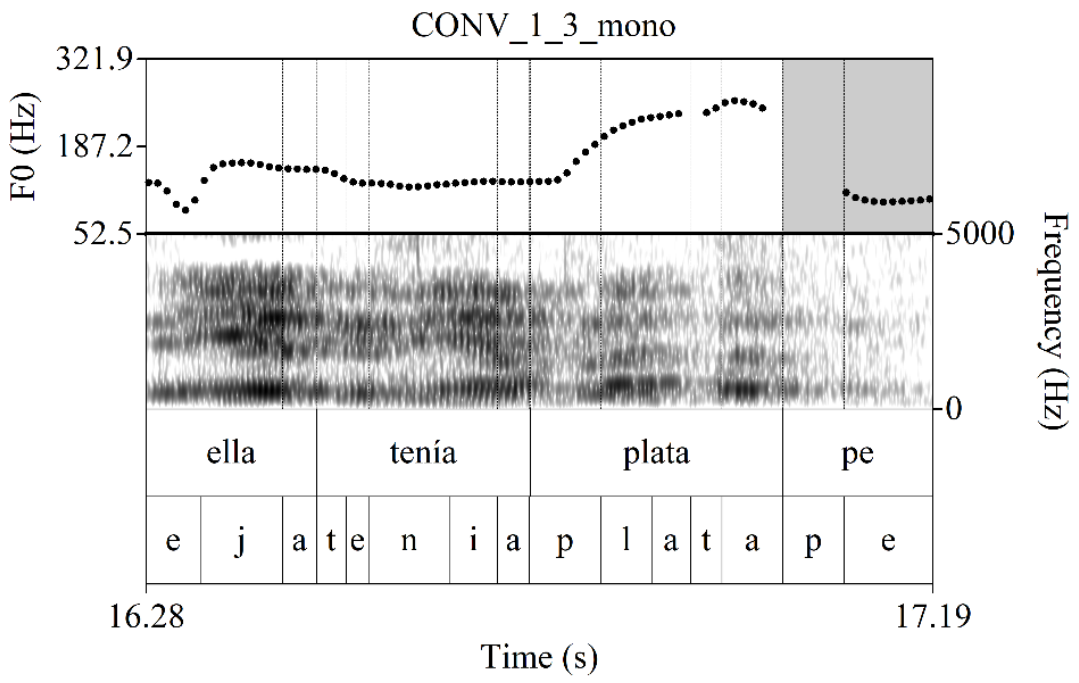


Figura 2. Contorno tonal de la frase Claro pe

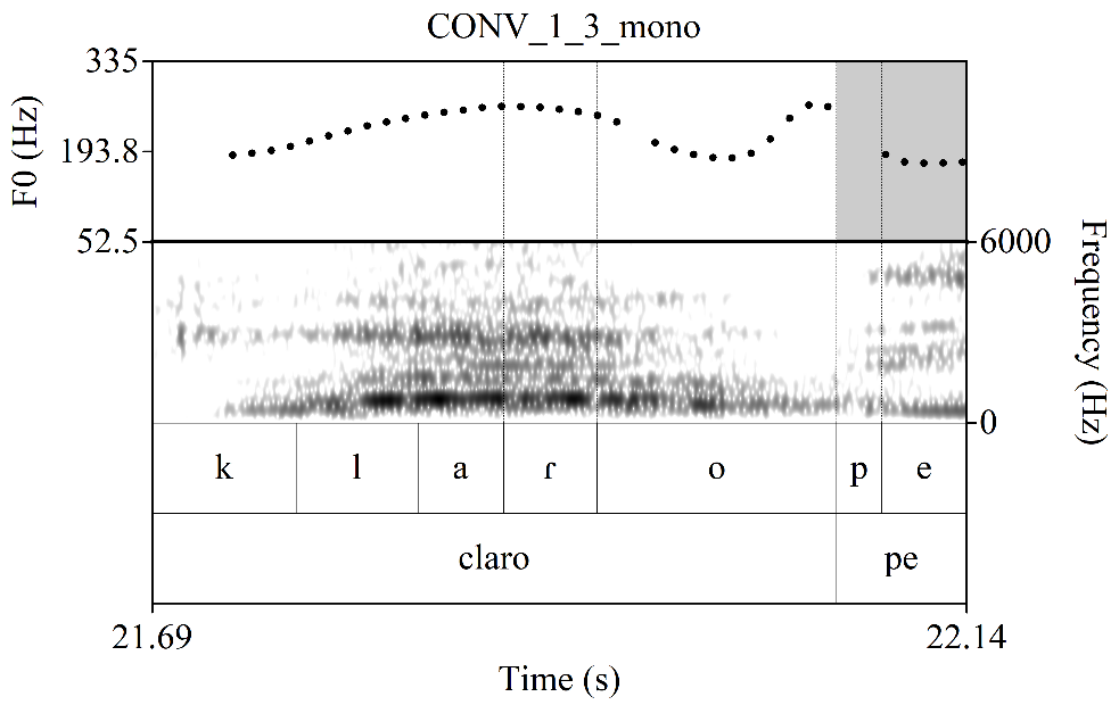

En (26) el dialogo se torna más narrativo, en el cual $\mathrm{R}$ cuenta una serie de hechos que vivió en su trabajo. Después de que indica que aparece el muchacho $\mathrm{R}$ incluye un dialogo, y para proseguir con su relato emplea «ya pues».

(26)

${ }_{1 R} 1$ y llegué a un pueblo otro pueblo otro pueblo llegué al destino $1 \mathrm{R} 1$ miro la hora a las cinco en punto

$1 \mathrm{R} 1$ entonces dije todavía está os, taba nublao pe bien oscuro estaba\$ $\mathrm{V}_{1}$

$1 \mathrm{R} 1$ entonces dije ah voa dormir pah

$1 \mathrm{R} 1$ y en es $\varnothing$ cinco y diez se aparece el muchacho

${ }_{1 R} 1$ ¡oe, yo te estao llamando!; no entra la llamada, ¿no?

$1 \mathrm{R} 1$ no entra -le digo pe- acá no entra movistar

${ }_{1} \mathrm{R} 1$ toy llamando -me dice- salí a ver el tractor y justo te encuentro acá

${ }_{1} \mathrm{R} 1$ ya pe me dice este que amanezca un poquito más para poder sacar $1 \mathrm{R} 1$ a las seis ya estaba clarito ya; lo sacó el esto,

$1 \mathrm{R} 1$ y seis y media hemos bajado pe 
En la figura 3 se aprecia un contorno tonal ascendente en el uso de la construcción «ya pues» con un valor continuativo. Se aprecia, además, los procesos fonético-fonológicos de elisión de /s/ y monoptongación.

Figura 3. Contorno tonal de la frase Ya pe me dice

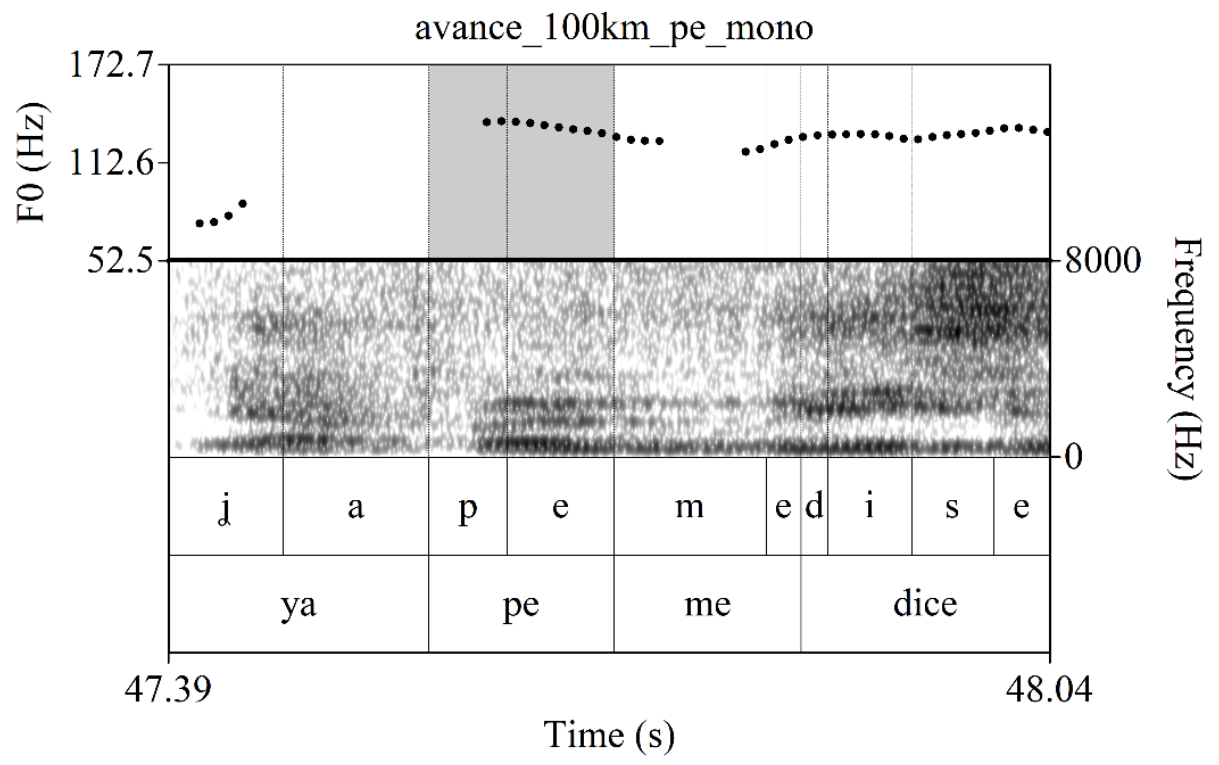

En suma, en las conversaciones espontáneas se aprecian discursos dialogados y narrativos. En los primeros, el uso de pues cumple las funciones aclarativa y enfática. En este caso, la partícula se ubica al final de la frase y presenta un tono descendente. Los procesos que sufre la palabra son dos: elisión de /s/ y monoptongación; pero el primero es el más frecuente. En los discursos narrativos, el pues, además de las funciones aclarativa y enfática, cumple las funciones continuativa e inferencial. En estos casos, la partícula presenta una posición intermedia y un tono ascendente. Hay una duración menor del pues. La construcción \{claro + pe\} cumple función aclarativa; \{pue/ pe\} cuando es enfática; $\{y a+$ pe $\}$ se emplea en funciones continuativa e inferencial. 
De la causalidad a la súplica: nuevas funciones del marcador pues

Tabla 2. Forma y función de pues en conversaciones espontáneas

\begin{tabular}{|c|c|c|c|c|}
\hline Función & Proceso & Posición & Tono & Duración \\
\hline Aclarativa & $\begin{array}{l}\text { Monoptongación } \\
4 / 4 \\
\text { Elisión de /s/ } \\
4 / 4\end{array}$ & Final & Bajo & 130 \\
\hline Enfática & $\begin{array}{l}\text { Monoptongación } \\
3 / 7 \\
\text { Elisión de /s/ } \\
6 / 7\end{array}$ & Final & Bajo & 183 \\
\hline Continuativa & $\begin{array}{l}\text { Monoptongación } \\
1 / 1 \\
\text { Elisión de /s/ } \\
1 / 1 \\
\text { Inserción de }\{y a\}\end{array}$ & Intermedia & Alto & 138 \\
\hline Inferencial & $\begin{array}{l}\text { Monoptongación } \\
1 / 1 \\
\text { Elisión de /s/ } \\
\text { 1/1 } \\
\text { Inserción de }\{y a\}\end{array}$ & Intermedia & Alto & 104 coe \\
\hline
\end{tabular}

\subsection{Entrevistas}

En las entrevistas semiestructuradas, además de las funciones vistas en las conversaciones espontáneas, como son las continuativas e inferenciales y las aclarativas y enfáticas, aparecen la función consecutiva y la de comentador. Con respecto a los parámetros fonéticos, la función continuativa presenta mayor duración; la función inferencial se expresa con un tono ascendente; los procesos fonético-fonológicos de debilitamiento del segmento /s/ o del diptongo /ue/ se restringen en la función enfática. 
Verónica Jenny Lazo García

Tabla 3. Forma y función de pues en entrevistas

\begin{tabular}{|c|c|c|c|c|}
\hline \multicolumn{5}{|c|}{ Entrevistas semiestructuradas } \\
\hline Función & Proceso & Posición & Tono & Duración \\
\hline Consecutiva & $\begin{array}{l}\text { Monoptongación } 1 / 3 \\
\text { Aspiración } \\
1 / 3\end{array}$ & Intermedia & Bajo & 270 \\
\hline Continuativa & $\begin{array}{l}\text { Aspiración } 1 / 4 \\
\text { Inserción }\{y\} 3 / 4 \\
\text { Inserción }\{y a\} 1 / 4\end{array}$ & Intermedia & Bajo & 403 \\
\hline Inferencial & $\begin{array}{l}\text { Inserción }\{y a\} 2 / 2 \\
\text { Sonorización de /p/ 1/2 }\end{array}$ & Intermedia & Alto & 309 \\
\hline Comentador & Inserción $\{\mathrm{MD}\}$ & Intermedia & Bajo & 311 \\
\hline Aclarativo & Elisión de /s/ 1/1 & Final & Bajo & 290 \\
\hline Enfático & $\begin{array}{l}\text { Monoptongación } 1 / 7 \\
\text { Debilitamiento de /s/3/7 }\end{array}$ & $\begin{array}{l}\text { Acompaña } \\
\text { al término }\end{array}$ & Variable & 290 \\
\hline
\end{tabular}

En 27 se aprecia un dialogo en el que se mencionan hechos: crear, hablar, comunicar, pedir. Pero para anticipar una idea importante, continuar el relato porque ha habido un inciso adicional se emplea la construcción «ya pues».

$(27)$

A: El inglés, solo cuando estoy en el Icpna pero curiosamente yo nunca había creado un grupo de whatsapp solo para hablar inglés (risas)

G:

[ya]

A:

Siempre creo

grupos de Whatsapp para comunicar sobre las tareas de inglés pues, pero nunca en inglés

G:

Yaaa

A:

[Pero no

sé] Me pidieron el número y ya pues y tuve que crear un grupo / y // todo se habla en inglés / como que si estuvieran practicando así que es muy raro para mí, soy la única que habla en español.

(COLOO2) 
Figura 4. Contorno tonal de la frase Ya vues continuativo

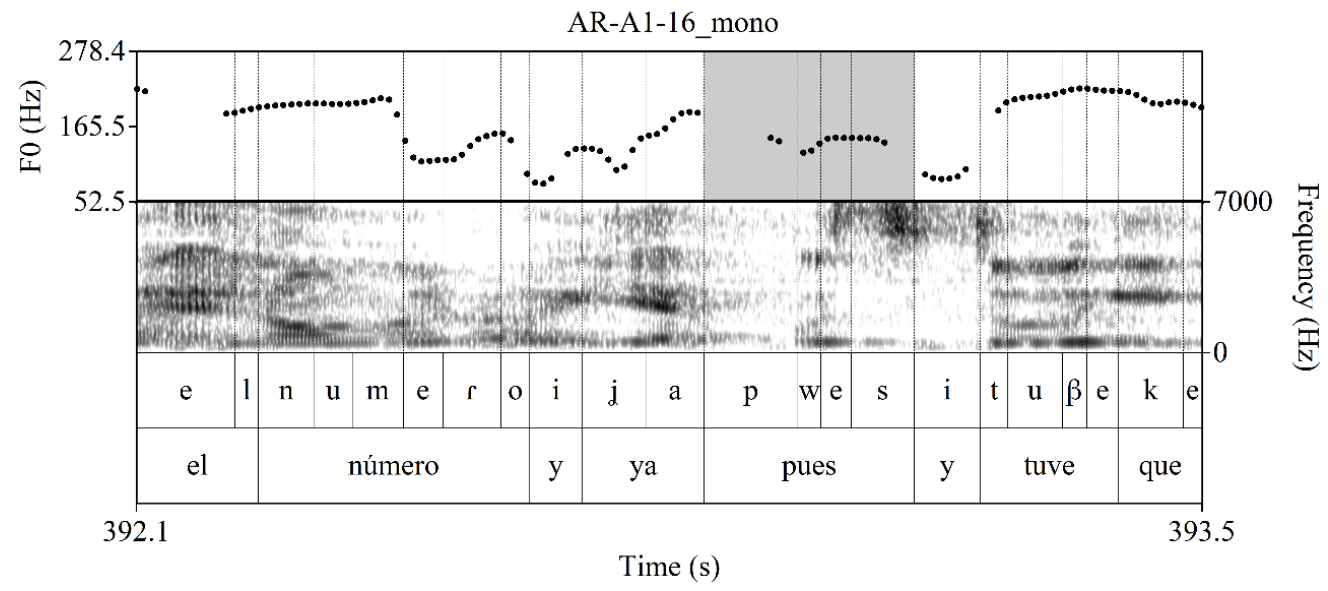

\subsection{Diálogos}

En esta sección se presenta diálogos creados por jóvenes estudiantes de primer año de la Universidad Agraria. En (28) la intervención reactiva de B expresa un matiz de desgano. En la figura 5 se aprecia el alargamiento de la construcción y el tono descendente.

(28)

Ii 1A1: Oe, Ñaño, vamo a la clase de lengua

Ir 1B1: Ya: pue

Figura 5. Contorno tonal de la frase Ya pue de desgano

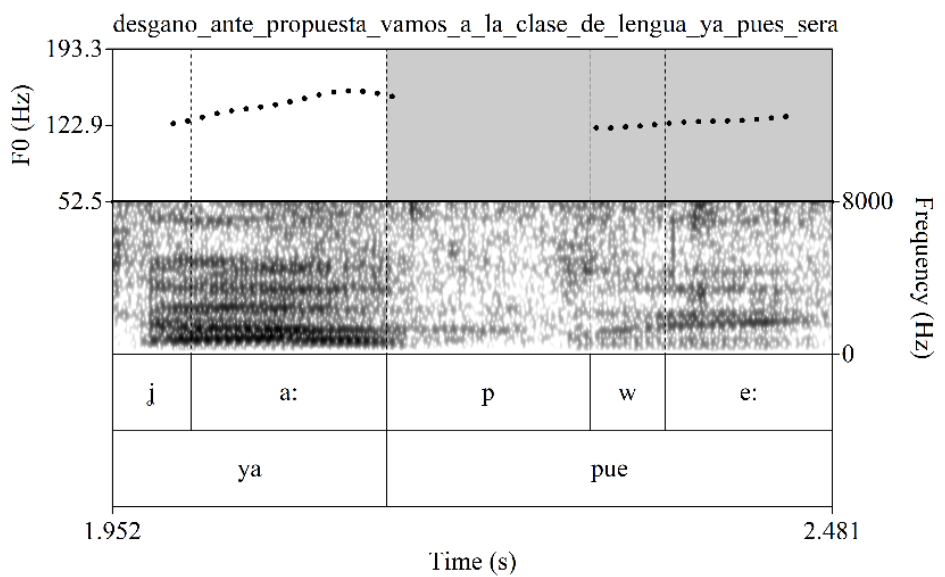


En (29) la interlocutora B reacciona de manera favorable a la invitación de A. La figura 6 muestra una articulación reforzada de la construcción «ya pues».

(29)

1A1: Oh, ahí está mi amiga Candy (corre y se acerca a Candy)

Ii 2A2: Candy, Candy, Candy, no sabes, ¡hoy va a haber un tonazo que va a estar de rompe. ¡Aparte, va a ir el chico que te gusta!

Ir 1B1: ¡Ya pues vamos!

Figura 6. Contorno tonal de la frase Ya pue de cordialidad

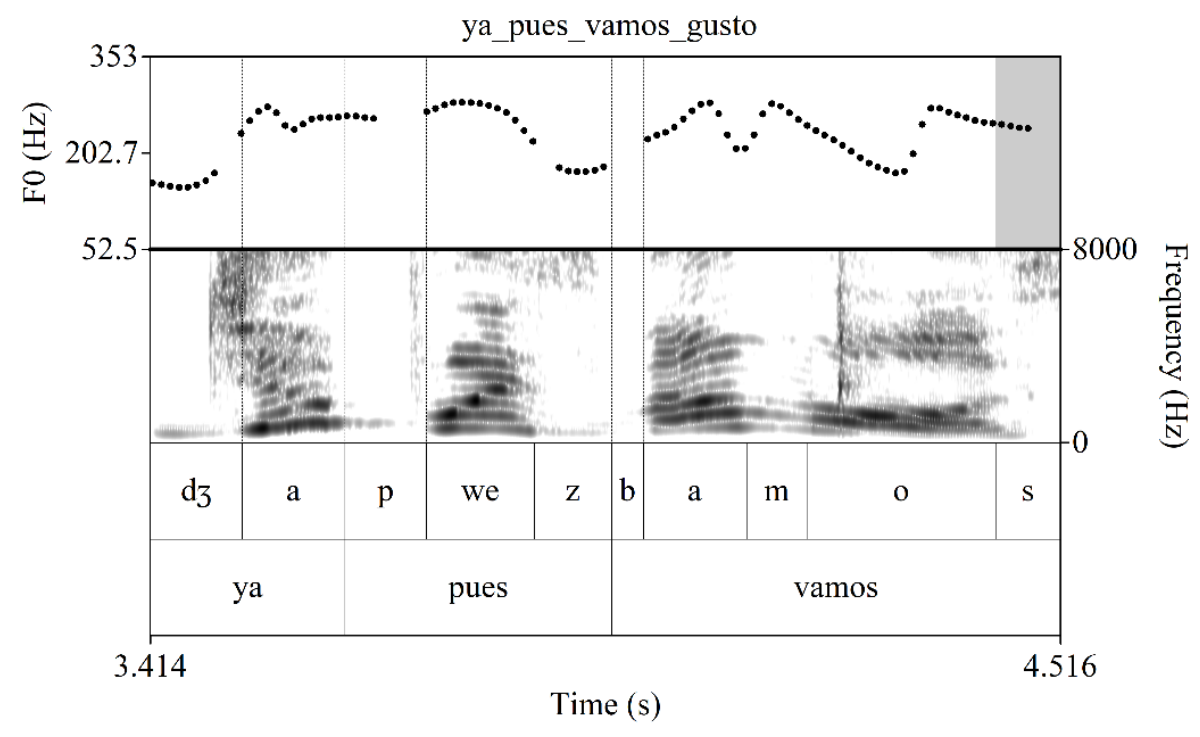

En (30) se aprecia un nuevo matiz de pues: el sarcasmo. Su posición es la inicial y se caracteriza por el alargamiento (v. Figura 7).

(30)

Ii 1A1: Hola Jorge, oye adivina, hoy día estudié para Matemática

Ir 1B1: ¡Ya pueees!, si tú nunca estudias (riendo)

Ir-i2A2: Oy, de verdad he estudiado pue, ¿no me crees?

Ir 3B3: No, no estudias... 
De la causalidad a la súplica: nuevas funciones del marcador pues

Figura 7. Contorno tonal de la frase Ya pue de sarcasmo

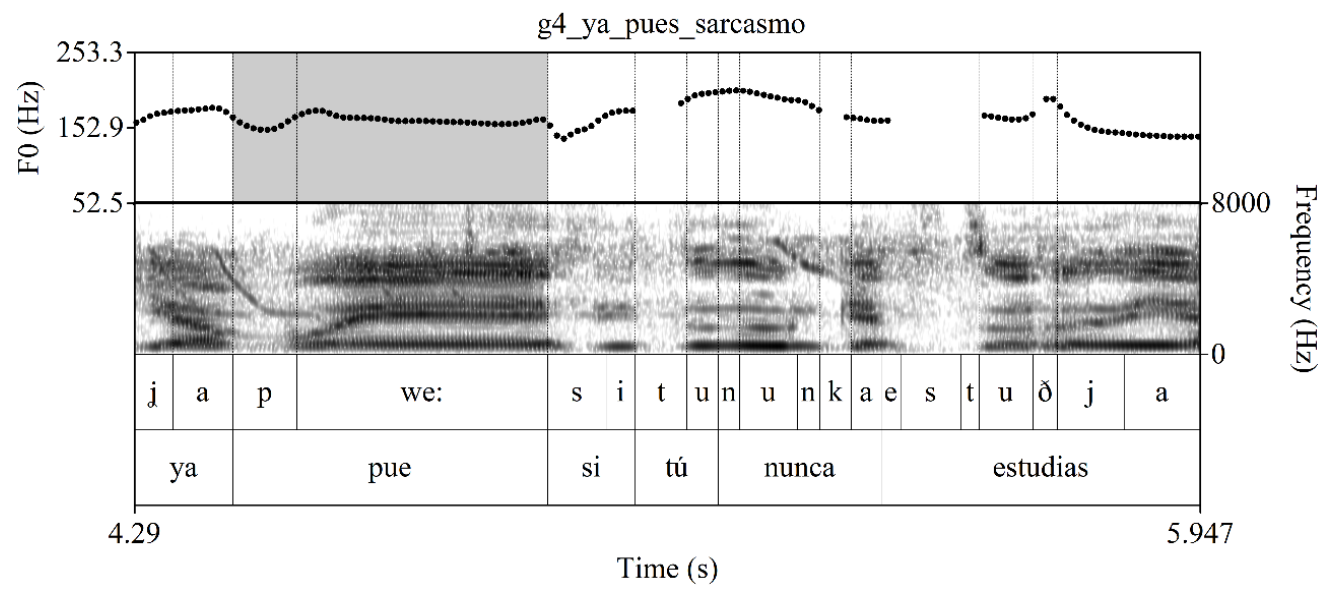

La partícula pues o la construcción ya pues pueden indicar súplica. En (31) A emplea la construcción «ya pues» para anticipar una súplica.

$(31)$

1A1: Profesora, nos puede dar una oportunidad, por favor, para volver a dar el examen.

1B1: Ay, no, no, no. Debieron estudiar antes del examen.

2A2: Ya pue, profesora. No sea así.

2B2: No, ya están puestas las notas.

Figura 8. Contorno tonal de la frase Ya pue de súplica

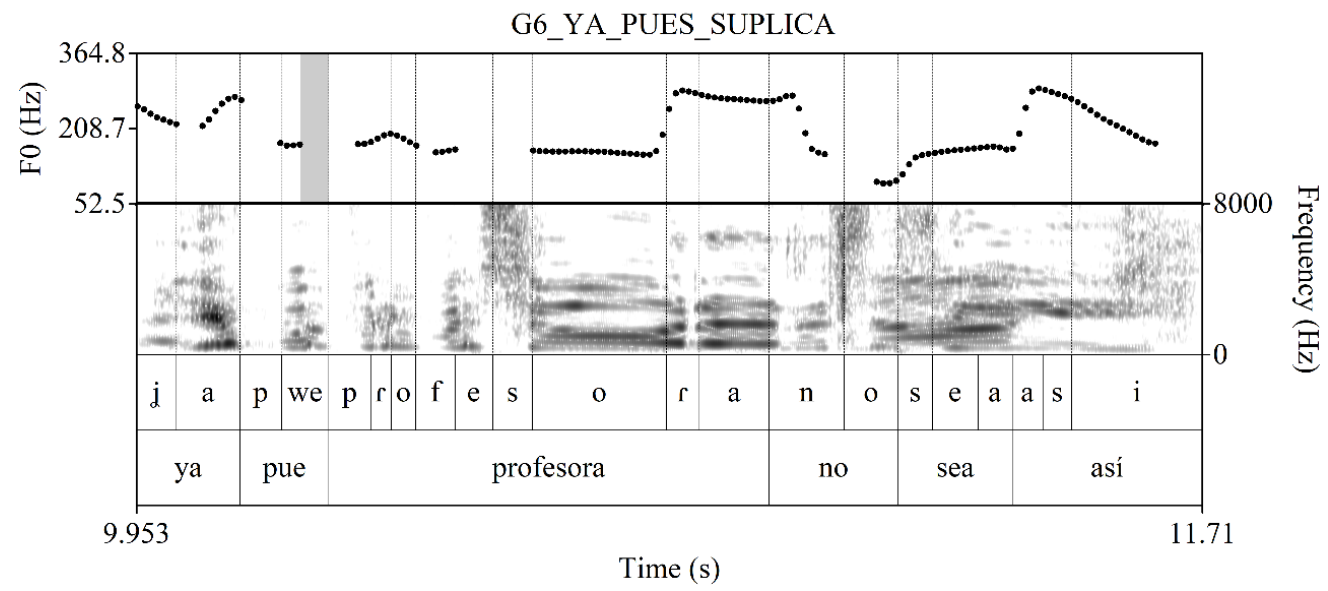


En los diálogos creados por los estudiantes, se aprecia dos funciones de pues: la de contacto y la de confirmación. Sin embargo, estas funciones son expresadas con matices pragmáticos nuevos. En la corroboración de la información, se aprecia valoración subjetiva de la información mediante la cortesía; es decir, la corroboración adquiere un matiz de desgano, de cordialidad y de sarcasmo. En la función de contacto, se aprecia el acto de habla directivo. En todos estos usos, se ha insertado el adverbio ya. Para indicar desgano, se añadió además el verbo ser en futuro del indicativo. El matiz de sarcasmo aparece con mayor duración debido a que se incurre en alargamiento vocálico en este contexto. El tono descendente caracteriza los matices subjetivos de sarcasmo y súplica. Esta última puede aparecer al inicio o al final del enunciado, y dura más cuando aparece al final.

\begin{tabular}{|c|c|c|c|c|c|}
\hline \multicolumn{6}{|c|}{ Diálogos semiestructurados } \\
\hline \multicolumn{2}{|c|}{ Función } & Proceso & Posición & Tono & Duración \\
\hline \multirow{3}{*}{ 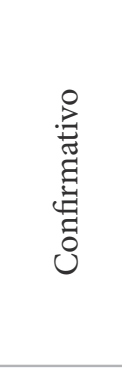 } & + cortesía & $\begin{array}{l}\text { Inserción de }\{y a\} \\
2 / 2\end{array}$ & Reactivo & Variable & 273 \\
\hline & -cortesía & $\begin{array}{l}\text { Inserción de }\{y a+\text { será }\} \\
2 / 2\end{array}$ & Reactivo & Variable & 334 \\
\hline & -credibilidad & $\begin{array}{l}\text { Inserción de }\{\text { ya }\} \\
3 / 3 \\
\text { Alargamiento vocálico } \\
3 / 3\end{array}$ & Reactivo & Bajo & 645 \\
\hline 节 & -exhortación & $\begin{array}{l}\text { Inserción de }\{y a\} \\
4 / 5\end{array}$ & $\begin{array}{l}\text { Inicial } 3 \\
\text { Final } 2\end{array}$ & Bajo & 421 \\
\hline
\end{tabular}

Los usos de pues dependen del contexto, así como del registro formal, semiformal o informal, y dentro de estos registros, depende de la intención y del género discursivo. Si bien se han reportado usos causales y consecutivos, en registros más formales, y metadiscursivos y de contacto en registros informales, estos usos dependen del instrumento de recolección de datos.

Si bien Dorta y Domínguez (2001) señalan que la sintaxis se ve enriquecida con estudios pragmáticos vía gramaticalización, pareciera que las funciones no corresponden únicamente a la partícula pues, sino a la estructura discursiva en la que aparece, al tono y a la duración. En ese mismo sentido, Uribe Mallarino (2005) señala que en expresiones formadas por pues, como pues bien y bueno pues, esta partícula se limita a reforzar el sentido de la palabra que acompaña. 
De la causalidad a la súplica: nuevas funciones del marcador pues

Por su parte, Villa Frey (2017) describe el uso de patrones distintos para la función confirmativa, como son sí pues, ya pues, claro pues, así pues, y cada uno aporta un matiz distinto de confirmación. Por tanto, creemos que en expresiones como ya pues, será pues, la partícula pues forma parte de una construcción fijada por el uso, con diferentes valores pragmáticos. Por lo tanto, y debido a que una construcción puede cumplir más de una función a la vez, establecer las funciones de pues depende de la estructura o de la construcción donde aparece, además de aspectos fonológicos, los cuales no se han desarrollado midiendo aspectos como tono, duración e intensidad, sino solo pausas.

Zavala (2006) propone una influencia del quechua en aquellos usos al final del discurso. Es necesario corroborar si el pues andino tiene características particulares, como la actitud e intención de hablante hacia el oyente en términos de información proporcionada o si el uso particular del pues en Lima es parte de los procesos de cambio del español mediante procesos pragmático-cognitivos.

En suma, se ha podido apreciar que, si bien sí se han propuesto los sentidos / funciones de pues, no se ha explicado la relación entre ellos. Además, como señala Villa Frey (2017), el tipo de función que se describa depende de la forma como se recogen los datos, a veces no hay frecuencia de uso de una función debido a que ese pues particular es más conversacional y el instrumento de recolección de datos no fue diseñado con ese criterio.

\section{Conclusiones}

1. Desde el punto de vista fonético-fonológico, generalmente las construcciones con pues presentan tono descendente, pero la función de narración, es decir, la continuativa e inferencial presenta tono ascendente. Cuando pues se usa como marcador de sarcasmo presenta mayor duración que en las otras construcciones. Se aprecia, además, procesos fonológicos de debilitamiento de /s/, monoptongación para las funciones aclarativa y enfática; el proceso de inserción de otros marcadores en las funciones continuativa, inferencial, de contacto y aclarativa con matices pragmáticos de desgano, cordialidad y sarcasmo.

2. En el registro espontáneo predomina la posición final de pues, que generalmente es de tipo enfático y aclarativo, y se pronuncia como [pe]. En el registro semiespontáneo (diálogos recreados), predomina la posición inicial y se emplea la construcción [ya pwes] y solo en este registro aparecen los reactivos con matices 
expresivos de súplica, sarcasmo y desgano. En la entrevista (registro semiformal), se observa mayor frecuencia de la posición intermedia, la cual es sobre todo argumentativa y corresponde a las construcciones [pweh] y [pwes].

3. La construcción «ya pues» cumple diversas funciones en el discurso. Puede enfatizar el elemento del discurso precedente, como una intervención reactiva; puede continuar un relato cuando previamente se ha presentado un inciso; puede adoptar matices pragmáticos de desgano, cordialidad, sarcasmo y súplica.

4. Las mujeres emplean pues en posición intermedia, mientras que los varones, en posición final. Además, las mujeres no pronunciaron [pe] en sus construcciones. La construcción \{claro pe\} solo fue pronunciada por varones.

\section{Bibliografía}

Alarcos, E. (1994). Gramática de la lengua española. Madrid : Espasa Calpe.

Bello, A. (1887). Gramática de la lengua castellana. Barcelona: EDAF.

Bosque, I. (2002). Las categorías gramaticales. Relaciones y diferencias. Madrid: Síntesis.

Calsamiglia, H., \& Tusón, A. (2001). Las cosas del decir. Manual de análisis del discurso. Barcelona: Ariel.

Calzado Roldán, A. (2015). Las construcciones en la clase de español como lengua extranjera. Marco ELE. Revista de Didáctica, 1-15.

Caravedo, R. (1990). Sociolingüística del español de Lima. Lima: Pontificia Universidad Católica del Perú.

Cortés, L., \& Camacho, M. M. (2005). Unidades de segmentación y marcadores del discurso. Madrid: Arco Libros.

Dorta , J., \& Domínguez , N. (2001). Polifuncionalidad discursiva y comportamiento prosódico prototípico del marcador pues. Español Actual, 45-53.

Escandel, M. (2006). Introducción a la pragmática. Barcelona: Ariel.

Garachana, M., \& Hilferty, J. (2005). ¿Gramática sin construcciones? Verba, 32, 385-396.

García-Miguel, J. (2005). Aproximacion empirica a la interacción de verbos y esquemas construccionales, ejemplificada con los verbos de percepción. ELUA. Estudios de Lingüistica de la Universidad de Alicante, 161-191.

Grajales Alzate, R. (2011). Funciones del marcador discursivo pues en el habla de Medellín, Colombia. Forma y Función, 24(1), 25-45.

160 Lengua y Sociedad 
De la causalidad a la súplica: nuevas funciones del marcador pues

Gras Manzano, P. (2010). Gramática en interacción: una propuesta desde la gramática de construcciones. . Linguistica e hispanismo. Lugo, 283-298.

Guevara, G. (2015). Funciones del marcador discursivo pues en un corpus oral. UPEL. Revista Multidisciplinaria Dialógica, 294-323 .

Martín Zorraquino , M. A., \& Portolés Lázaro, J. (1999). Los marcadores del discurso. En Gramática descriptiva de la lengua española. Entre la oración y el discurso. Morfología (pp. 4051-4214). Madrid: Espasa Calpe.

Portolés, J. (2001). Marcadores del discurso. Barcelona: Ariel.

Sanchez, A. (2015). Migraciones internas en el Perú. Lima: oIM.

Uribe Mallarino, M. (2005). Conectores y contrastividad: el caso de pues. AISPI. Actas XXIII (págs. 563-578). Centro Virtual Cervantes.

Van Dijk, T. (2016). Estudios críticos del discurso: un enfoque sociocognitivo. Discurso y Sociedad, 137-162.

Zavala, V. (2006). Transferencia de funciones evidenciales del quechua: el rol de pues como marcador discursivo en el español andino. Lexis, 3o(1), 55-82. 